\title{
Comparison of Routine Care Versus Transcutaneous Electrical Nerve Stimulation (TENS) for Treatment of Back Pain in the ED \\ Yanina Purim-Shem-Tovi*, Jean-Philippe Daniel ${ }^{2}$, Kevin Dyer $^{2}$, Michael Lavin $^{3}$, Nicholas Chien ${ }^{4}$, Shital Shah ${ }^{1}$
}

${ }^{1}$ Rush University Medical Center, Chicago, IL, USA.

${ }^{2}$ Medical Student, Rush University Medical Center, Chicago, IL, USA

${ }^{3}$ Medical Student, Sackler School of Medicine, Tel Aviv, Israel .

${ }^{4}$ Resident, Emergency Medicine, Rush University Medical Center, Chicago, IL, USA.

*Corresponding Author: Yanina Purim-Shem-Tov, MD, MS, FACEP Rush University Medical Center 1653 W. Congress Parkway Chicago, IL, USA.

\section{Abstract}

INTRODUCTION: There are limitations to acute medical management oflow back pain in the ED. Transcutaneous electrical nerve stimulation (TENS) provides a non-invasive, safe, accessible, and promising therapy.

OBJECTIVES: To evaluate the role of a TENS unit in managing low back pain in the ED, and to compare the average patient length of stay in the ED to conventional treatment.

METHODS: 71 patients with a chief complaint of low back pain were enrolled in the active arm. Pain scores on a 0-10 scale were obtained before and after treatment with the TENS units. The control group included 70 historical cases with conventional treatment. T-test analysis was used to evaluate for any statistical difference in pain reduction.

RESULTS: The pain scale before and after treatment was statistically significant between control and active arms: Before--controls $8.53 \pm 1.52$ and active arm $7.65 \pm 1.81$; after-- controls $5.89 \pm 2$ and active arm (5.01 $\pm 2.65)$. The Delta score related variables were not statistically significant between historical and treatment groups. An analysis was conducted for the EXACT matched pairs $(n=25)$. The pain scale before treatment was statistically significant between historical arm ( $8.52 \pm 1.45)$ and active arm $(7.56 \pm 1.83)$. The pain scale after treatment was NOT statistically significant between historical and active arms. The delta score related variables were NOT statistically significant between the two arms. Length of stay was not statistically significant between the arms.

CONCLUSION: These results suggest that TENS is a viable treatment modality for lower back pain in the ED.

Keywords: back pain, transcutaneous electrical nerve stimulation, TENS, ED, therapy.

\section{INTRODUCTION}

Low back pain is a common, debilitating, and costly condition. It is the fifth most common reason for physician evaluation with an annual prevalence of $15 \%$ to $20 \% .{ }^{1}$ Beyond the costs of medical management, patients with back pain often incur further losses in the workplace due to productivity time lost and compensation costs. ${ }^{1}$ Low back pain comprises $4.39 \%$ of adult patient visits to the emergency department and is one of the top ten presenting chief complaints. ${ }^{2}$ Due to the high prevalence, it is important for physicians to understand potential treatment options for low back pain.

The severity of the symptoms associated with acute and chronic back pain often drives treatment, which includes non-opioid analgesics, opioids, physical 
therapy, cold or heat therapy, nerve therapy, epidural corticosteroid injections, behavioral therapy, spinal cord stimulation, and acupuncture. ${ }^{3}$ Traditionally, routine care is performed by providing nonsteroidal anti-inflammatory drugs (NSAIDs), opioids, benzodiazepines, and/or muscle relaxants, in addition to advising patients to remain active. ${ }^{4}$ These therapies must be individualized due to side effects and to prevent drug-drug interactions. NSAIDs have shown efficacy as a treatment for low back pain; however, its use is limited by concentration and interval of dosing along with multiple effects including cardiovascular side effects, gastrointestinal bleeding, and NSAID induced nephrotoxicity. ${ }^{27}$ Opioid's and benzodiazepines can be used for short-term analgesia but chronic use is complicated by tolerance and dependency. ${ }^{6}$ Muscle relaxants vary by mechanisms of action and side effect profiles. The limitations of current treatments, along with their associated side effects, call for the need for a safer, more accessible, and clinically effective treatment for low back pain.

Transcutaneous electrical nerve stimulation (TENS) is a non-invasive therapy that has shown promising results in the management of low back pain. TENS units utilize skin surface electrodes that provide electrical stimulation to peripheral nerves, typically at the site of injury. ${ }^{4}$ The electrical current activates muscarinic, opioid and serotonin receptors centrally, as well as $\alpha$-2-noradrenergic and opioid receptors peripherally, resulting in analgesia. ${ }^{7}$ It is also believed that TENS activates large diameter nocireceptive afferent fibers to reduces pain signaling thereby decreased pain perception ${ }^{7}$. Because TENS is non-pharmacological and site-specific, it is an ideal method for localized pain relief without the concern for medication interaction or side effects. These devices are also accessible overthe-counter from most pharmacies.

Several studies have investigated the potential efficacy of TENS as a treatment for low back pain. A recent study showed that utilizing TENS for treatment of chronic low back pain resulted in a significant reduction in the number of oral analgesic, fewer per-patient opioid costs, and decreased pharmacy use compared to control groups. ${ }^{8}$ In contrast, some studies have shown limited functionality of TENS as a treatment for acute and chronic back pain. ${ }^{5,9}$ More specifically, those reviews and analyses that looked at TENS unit treatment for back pain had results ranging from not recommended to effective for pain control. However, the studies included in the reviews had differing inclusion/exclusion criteria, inconsistent dosing of TENs unit, and varying patient populations and varying mechanisms of back pain (i.e. MSK vs nerve damage such as multiple sclerosis). Other factors that can influence measured efficacy include stimulation parameters such as intensity, frequency, duration of treatment, comorbidities, demographics, device sophistication and position of stimulation. ${ }^{10,11}$ Therefore, further research with a more homogenous patient sample with consistent pain physiology and consistent dosing of the TENS unit is needed to understand the viability of this technology as a major tool for back pain relief.

\section{METHODS}

The non-inferiority study used a convenience sample of patients presenting to the emergency department (ED) with a chief complaint of lower back pain. The study was conducted at a large urban academic inner city hospital ED from July 2018 to October 2019. A total of 70 patients were placed into the treatment arm and 70 historical case controls were used for the control arm. The team calculated that approximately 61 patients would need to be enrolled in each group to observe appropriate effect. The decision was made to compare two groups of 70 patients each in order to have a power of $\sim 0.85$. Inclusion criteria consisted of age over 18, English-speaking, and presentation to the emergency department with a chief complaint of or multiple complaints including back pain. Patients with both acute and acute-on-chronic back pain were included. Exclusion criteria included current status of breastfeeding, pregnancy or chance of being pregnant, history of seizures, any type of electrical implant or pacemaker, diagnosis of an arrhythmia, abnormal skin on the lower back (rash, cancerous lesion, etc.), history of cancer, concern for cauda equina, inability to provide appropriate informed consent as assessed by provider, or if they were admitted to the hospital. The historical arm was obtained through an internal database of patients who presented to the Emergency Department with acute or acute-on-chronic back pain during the year of the study's proposal. Patients fitting the study's inclusion and exclusion criteria were identified, and 70 patients were randomly selected from the database. The pain scores pre-treatment and 30 minutes post-treatment were available in the chart. 
Comparison of Routine Care Versus Transcutaneous Electrical Nerve Stimulation (TENS) for Treatment of Back Pain in the ED

The dependent variables consisted of the patient pain scores before and after receiving treatment, and length of stay in the ED. This study was approved by the Institutional Review Board, and was supported by departmental self-funding.

Prior to treatment, patients rated their current level of pain on a scale of 1-10 and the patient was asked to point to the exact location of their pain. An over-thecounter Aleve Direct Therapy TENS unit was placed by a research assistant at the spinal level closest to the patient's pain. The device consists of two electrodes covered by adhesive gel pads and a separate remote used to increase or decrease stimulation intensity. The non-adhesive center was placed over the patient's midline spine while the two adhesive pads were placed over the paraspinal muscles. Patients received 30 minutes of treatment consisting of three stages: 5 minutes of high frequency stimulation (pulse duration $120 \mu \mathrm{s}$, frequency $80-120 \mathrm{~Hz}$ ), 20 minutes of low frequency stimulation (pulse duration $240 \mu \mathrm{s}$, frequency $5-10 \mathrm{~Hz}$ ), and 5 minutes of high frequency stimulation (pulse duration $120 \mu \mathrm{s}$, frequency 80-120 $\mathrm{Hz}$ ). After 30 minutes of TENS therapy, the device was removed and a post-treatment pain score was obtained on a 1-10 scale. If there was incomplete pain control as defined as a post-treatment pain level $\geq 5$ on the $0-10$ scale, then standard therapies were Table1. Patient Demographics and Comorbidities.

\begin{tabular}{|l|l|l|}
\hline & Active Arm & Historical Arm \\
\hline & 69 & 70 \\
\hline Male & $28(41 \%)$ & $37(53 \%)$ \\
\hline Female & $41(59 \%)$ & $33(47 \%)$ \\
\hline Age 18-29 & $14(20 \%)$ & $21(30 \%)$ \\
\hline Age 30-39 & $17(25 \%)$ & $19(27 \%)$ \\
\hline Age 40-49 & $14(20 \%)$ & $13(19 \%)$ \\
\hline Age $>50$ & $24(35 \%)$ & $17(24 \%)$ \\
\hline Race: Black & $40(58 \%)$ & $42(60 \%)$ \\
\hline Race: White & $8(12 \%)$ & $10(14 \%)$ \\
\hline Race: Hispanic & $18(26 \%)$ & $11(16 \%)$ \\
\hline Race: Asian & $1(1 \%)$ & $2(3 \%)$ \\
\hline Race: Other & $2(3 \%)$ & $5(7 \%)$ \\
\hline Symptoms present 1-6 days & $41(59 \%)$ & $35(50 \%)$ \\
\hline Symptoms present 7-20 days & $9(13 \%)$ & $11(16 \%)$ \\
\hline Symptoms present $>20$ days & $19(28 \%)$ & $24(34 \%)$ \\
\hline Comorbidities & & \\
\hline Hypertension & $15(22 \%)$ & $19(27 \%)$ \\
\hline Diabetes & $10(14 \%)$ & $8(11 \%)$ \\
\hline Congestive Heart Failure & $1(1 \%)$ & $1(1 \%)$ \\
\hline Arthritis & $3(4 \%)$ & $3(4 \%)$ \\
\hline Other comorbidities $(<5)$ & $32(46 \%)$ & $41(59 \%)$ \\
\hline Other comorbidities $(\geq 5)$ & $11(16 \%)$ & $4(6 \%)$ \\
\hline
\end{tabular}

Descriptive demographics and comorbidities of patients enrolled into an active and control (historical) arms.

Archives of Emergency Medicine and Intensive Care V4 . I1 . 2021 
Comparison of Routine Care Versus Transcutaneous Electrical Nerve Stimulation (TENS) for Treatment of Back Pain in the ED

We analyzed active and historical arms for pain scale analysis, utilizing a standard 1-10 score. Initially, sample t-test was utilized for the unmatched pair's analysis. The pain scale before treatment was statistically significant between historical arm (controls) ( $8.53 \pm 1.52)$ and active arm $(7.65 \pm 1.81)$ groups. The pain scale after

Table2. Unmatched pairs analysis treatment was statistically significant between historical arm (5.89 \pm 2$)$ and active arm (5.01 \pm $2.65)$ groups. The Delta score related variables were not statistically significant between historical and treatment arm groups. This indicates that the historical and treatment arms had a similar reduction in pain scores. (Table 2)

\begin{tabular}{|l|l|l|l|}
\hline All Data & Control/Pre (n=70) & Arm (n=69) & T- Test \\
\hline Variables & $8.53 \pm 1.52$ & $7.65 \pm 1.81$ & $\mathrm{t}=3.09 \mathrm{df}=137 \mathrm{p}=0.002$ \\
\hline Pain scale before treatment & $5.89 \pm 2$ & $5.01 \pm 2.65$ & $\mathrm{t}=2.2 \mathrm{df}=126.7 \mathrm{p}=0.029$ \\
\hline Pain scale after 30 min of treatment & $2.64 \pm 1.64$ & $2.64 \pm 2.02$ & $\mathrm{t}=-0.01 \mathrm{df}=137 \mathrm{p}=0.995$ \\
\hline Delta Pain score & $0.31 \pm 0.2$ & $0.37 \pm 0.29$ & $\mathrm{t}=-1.26 \mathrm{df}=120.36 \mathrm{p}=0.209$ \\
\hline Delta Percentage Pain score & $0.69 \pm 0.2$ & $0.63 \pm 0.29$ & $\mathrm{t}=1.26 \mathrm{df}=120.36 \mathrm{p}=0.209$ \\
\hline Ratio Pain score & $208.41 \pm 117.72$ & $228.35 \pm 166.55$ & $\mathrm{t}=-0.82 \mathrm{df}=137 \mathrm{p}=0.416$ \\
\hline LOS Minutes &
\end{tabular}

$\mathrm{T}$ test analysis of patients treatment with TENS unit vs historical arms. Furthermore, the length of stay (LOS) in the ED had no statistical significance in the two groups.

We further conducted a propensity match pairing for comorbidities, race, age, and gender. (Table 3) This analysis was performed in order to remove any bias between the two arms of the study. Given the non-

inferiority design of the study, propensity matching of pairs allows us to compare patients in both arms that have similar features by demographics and comorbidities.

Table3. Propensity matches pairing.

\begin{tabular}{|l|l|l|l|}
\hline Matching Results - Samples included in the Analysis & Cumulative \% \\
\hline Matching & N & $\%$ & C \\
\hline Comorbidities + Race + Age + Gender (EXACT MATCH) & 25 & $41.7 \%$ & $41.7 \%$ \\
\hline Race + Age + Gender & 20 & $33.3 \%$ & $75.0 \%$ \\
\hline Age + Gender & 12 & $20.0 \%$ & $95.0 \%$ \\
\hline Gender & 3 & $5.0 \%$ & $100.0 \%$ \\
\hline
\end{tabular}

Determination of matching pairs by different variables

For the ALL matched pairs $(n=60)$ we used dependent t-test analysis. The pain scale before treatment was statistically significant between historical (8.57 \pm 1.54$)$ and active arm (7.78 \pm $1.77)$ groups. The pain scale after treatment was

Table4. Pain scores analysis for matched pairs. statistically significant between historical arm $(5.78 \pm 2)$ and treatment arm $(4.96 \pm 2.68)$ groups. However, the Delta score related variables were not statistically significant between historical and active arm groups. (Table 4)

\begin{tabular}{|l|l|l|l|l|}
\hline Matched Data & Control/Pre $\mathbf{( n = 6 0 )}$ & Arm $(\mathbf{n = 6 0})$ & T- Test & Correlation \\
\hline Pain scale before treatment & $8.57 \pm 1.54$ & $7.78 \pm 1.77$ & $\mathrm{t}=-2.61 \mathrm{df}=59 \mathrm{p}=0.011$ & $\mathrm{r}=0.02 \mathrm{p}=0.874$ \\
\hline $\begin{array}{l}\text { Pain scale after 30 min of } \\
\text { treatment }\end{array}$ & $5.78 \pm 2$ & $4.96 \pm 2.68$ & $\mathrm{t}=-2.1 \mathrm{df}=59 \mathrm{p}=0.04$ & $\mathrm{r}=0.18 \mathrm{p}=0.176$ \\
\hline Delta Pain score & $2.78 \pm 1.65$ & $2.83 \pm 2.06$ & $\mathrm{t}=0.13 \mathrm{df}=59 \mathrm{p}=0.898$ & $\mathrm{r}=0.1 \mathrm{p}=0.43$ \\
\hline Delta Percentage Pain score & $0.33 \pm 0.2$ & $0.39 \pm 0.3$ & $\mathrm{t}=1.4 \mathrm{df}=59 \mathrm{p}=0.168$ & $\mathrm{r}=0.17 \mathrm{p}=0.198$ \\
\hline Ratio Pain score & $0.67 \pm 0.2$ & $0.61 \pm 0.3$ & $\mathrm{t}=-1.4 \mathrm{df}=59 \mathrm{p}=0.168$ & $\mathrm{r}=0.17 \mathrm{p}=0.198$ \\
\hline LOS Minutes & $206.1 \pm 118.33$ & $\begin{array}{l}235.7 \pm \\
174.43\end{array}$ & $\mathrm{t}=1.14 \mathrm{df}=59 \mathrm{p}=0.257$ & $\mathrm{r}=0.1 \mathrm{p}=0.429$ \\
\hline
\end{tabular}

T test analysis for matched pairs between treatment (TENS) arm and historical arm. 
Comparison of Routine Care Versus Transcutaneous Electrical Nerve Stimulation (TENS) for Treatment of Back Pain in the ED

This indicates that the historical and active arms had similar reduction in pain scores. Furthermore, for matched pairs, the LOS was not statistically significant.

A separate analysis was conducted for the EXACT matched pairs $(n=25)$, using a dependent t-test. The pain scale before treatment was statistically Table5. Analysis of 25 Exact Matched Pairs. significant between historical arm $(8.52 \pm 1.45)$ and active arm (7.56 \pm 1.83$)$ groups. The pain scale after treatment was not statistically significant between historical arm and active arm groups. Furthermore, the delta score related variables were not statistically significant between historical and active arm groups. (Table 5)

\begin{tabular}{|l|l|l|l|l|}
\hline Exact Matched Data & $\begin{array}{l}\text { Control/ } \\
\text { Pre }(\mathbf{n}=\mathbf{2 5})\end{array}$ & $\begin{array}{l}\text { Arm } \\
(\mathbf{n}=\mathbf{2 5})\end{array}$ & $\mathrm{T}$ - Test & Correlation \\
\hline Pain scale before treatment & $8.52 \pm 1.45$ & $7.56 \pm 1.83$ & $\mathrm{t}=-2.17 \mathrm{df}=24 \mathrm{p}=0.04$ & $\begin{array}{l}\mathrm{r}=0.11 \mathrm{p}= \\
0.614\end{array}$ \\
\hline $\begin{array}{l}\text { Pain scale after 30 min of } \\
\text { treatment }\end{array}$ & $5.6 \pm 2.43$ & $4.72 \pm 2.81$ & $\mathrm{t}=-1.44 \mathrm{df}=24 \mathrm{p}=0.163$ & $\begin{array}{l}\mathrm{r}=0.32 \mathrm{p}= \\
0.113\end{array}$ \\
\hline Delta Pain score & $2.92 \pm 1.82$ & $2.84 \pm 2.53$ & $\mathrm{t}=-0.14 \mathrm{df}=24 \mathrm{p}=0.894$ & $\begin{array}{l}\mathrm{r}=0.11 \mathrm{p}= \\
0.616\end{array}$ \\
\hline Delta Percentage Pain score & $0.36 \pm 0.24$ & $0.39 \pm 0.33$ & $\mathrm{t}=0.45 \mathrm{df}=24 \mathrm{p}=0.658$ & $\begin{array}{l}\mathrm{r}=0.29 \mathrm{p}= \\
0.167\end{array}$ \\
\hline Ratio Pain score & $0.64 \pm 0.24$ & $0.61 \pm 0.33$ & $\mathrm{t}=-0.45 \mathrm{df}=24 \mathrm{p}=0.658$ & $\begin{array}{l}\mathrm{r}=0.29 \mathrm{p}= \\
0.167\end{array}$ \\
\hline LOS Minutes & $218.48 \pm$ & $\begin{array}{l}204.96 \pm \\
80.37\end{array}$ & $\mathrm{t}=-0.53 \mathrm{df}=24 \mathrm{p}=0.602$ & $\begin{array}{l}\mathrm{r}=0.19 \mathrm{p}= \\
0.354\end{array}$ \\
\hline
\end{tabular}

T test analysis for the 25 exact matched pairs between the treatment (TENS) and historical arms.

This indicates that the historical and active arms have similar drop in pain score. For EXACT matched pairs, LOS was also not statistically significant.

\section{DISCUSSION}

According to the National Hospital Ambulatory Medical Care Survey from 2017, emergency departments (EDs) treat over 3 million people annually with a complaint of back pain. ${ }^{12}$ Classes of medications most frequently used to treat back pain are opioids, followed by non-steroidal anti-inflammatory drugs, and muscle relaxants. ${ }^{13}$ National Drug abuse database reports drug overdose deaths involving prescription opioids rose from 3,442 in 1999 to 17,029 in $2017 . .^{14}$ Multiple national organizations, including American Medical Association (AMA), American Hospital Association (AHA), American College of Emergency Physicians (ACEP) and other organizations have recently implemented Alternatives to Opioid (ALTO) programs in many EDs across the country ${ }^{18}$ In general, ALTO is a multimodal approach to pain management aimed at regulating multiple receptors to achieve pain control. ${ }^{19}$ The first ED ALTO program was launched in January 2016 at St. Joseph's Healthcare System in New Jersey and provided alternative protocols for pain management such as nitrous oxide, trigger point injections, and ultrasound guided nerve blocks. ${ }^{19}$

Various non-pharmacologic modalities have been recommended for the management and treatment of low back pain. Physical therapy is one treatment option focused on continued exercise geared towards flexibility and strength to improve pain and function. ${ }^{21}$ While other non-pharmacologic modalities such as heat therapy, manipulation, acupuncture have been validated as effective therapies in the management of back pain, the use of transcutaneous electrical nerve stimulation (TENS) has yet to be fully characterized and there exists a need for higher quality trials..$^{22-24}$ A review of systematic reviews and meta-analyses between 2007-2014 showed mixed results on the efficacy of TENS for back pain. The use of inconsistent treatment protocols, complex etiologies of pain (cancer pain, post-op pain, labor pain, multiple sclerosis), differences in inclusion/exclusion criteria, and variation in patient populations likely contributed to differences in efficacy.

In the outpatient setting, electrical stimulation is one of the commonly utilized modalities to help with low back pain, primarily for chronic low back pain. TENS units are high frequency stimulators. There are 2 proposed mechanisms of action: local release 
of neurotransmitters (serotonin to block the pain signals), and dorsal column of the spinal cord inhibition of c-fiber nociception. Literature also suggests that electrical stimulation improves microcirculation and blood flow, which promotes healing. ${ }^{25}$ Because these devices are small and portable, patients can wear the device for extended periods of time. They are also easily accessible and can be purchased overthe-counter. Improved pain control results in better mobility and improved functionality for patients. In a recently study by Grover, et. al., the authors found that TENS units are a feasible modality to be used in the ED for pain management. ${ }^{26}$ Furthermore, the authors focused on the question of efficacy of this modality if added to standard therapy. Our study, which focuses on acute low back pain in the emergency department setting, demonstrates that TENS units can provide the same level of pain reduction as standard therapy.

Another important aspect of ED operations is patient throughput. While our data shows that patient throughput did not statistically differ for the treatment and historical arms, future research may seek to further delineate the possibility of increasing patient throughput. We have provided further support to implement this device in the ED as part of routine pain management, at least for patients who present with lower back pain and do not have any red flags signs or symptoms.

\section{LIMITATIONS}

This non-inferior study was a case-control, convenient sample conducted in an urban inner city academic hospital. Although we did find propensity matched pairs for various demographics and comorbidities, we did not find all 70 pairs. This study was not a randomized controlled trial. This study showed noninferiority in acute pain reduction, but ongoing pain after removal of the TENS device and after the patient left the emergency department was never followed. Manufacturer guidelines disclose that the device can safely be used multiple times throughout the day and recommend a minimum of 30 minutes between treatments. Future studies could potentially investigate a longer follow-up and repeated treatment. 24 patients who had taken at least one pain medication (e.g. NSAIDs, opioids, muscle relaxers, benzodiazepines, or lidocaine patch) $\geq 30$ minutes prior to enrollment in the study were flagged as they may have benefited from adjunctive treatment. We also did not study the logistical aspect of having TENS units available in the ED. For instance, would it be physical therapist providing such treatment as part of a consult? Some of the questions to explore: how to set up and order TENS unit treatment for coding and billing purposes, and is there reimbursement for such treatment in EM care? Further limitation of this study is that it was conducted in one Academic Urban Inner City hospital. Generalization of findings would be more applicable after having this conducted at different type of facilities with different patient populations.

\section{ConCLUSIONS}

This non-randomized case-control convenient sample study, we have shown that TENS units are an alternative and possible adjunctive method for treatment of patient with lower back pain presenting to the ED. This type of treatment does not increase patient length of stay. Not only is this a feasible modality, but it shows similar pain reduction as standard of care treatment. Further studies would need to be conducted to evaluate the logistical aspect of utilizing TENS units in the ED, assess long-term pain control, and utilization of TENS after patients leave the emergency department.

\section{REFERENCES}

[1] Atlas SJ, Deyo RA. Evaluating and managing acute low back pain in the primary care setting. $J$ Gen Intern Med. 2001 Feb;16(2):120-31. doi: 10.1111/j.1525-1497.2001.91141.x. PMID: 11251764; PMCID: PMC1495170.

[2] Edwards J, Hayden J, Asbridge M, Gregoire B, Magee K. Prevalence of low back pain in emergencysettings: asystematicreviewandmetaanalysis. BMC Musculoskelet Disord. 2017 Apr 4;18(1):143. doi: 10.1186/s12891-017-1511-7. PMID: 28376873; PMCID: PMC5379602.

[3] McIntosh G, Hall H. Low back pain (acute). BMJ Clin Evid. 2011 May 9;2011:1102. PMID: 21549023; PMCID: PMC3217769.

[4] van Tulder MW, Koes B, Malmivaara A. Outcome of non-invasive treatment modalities on back pain: an evidence-based review. Eur Spine J. 2006 Jan;15 Suppl 1(Suppl 1):S64-81. doi: 10.1007/ s00586-005-1048-6. Epub 2005 Dec 1. PMID: 16320031; PMCID: PMC3454555.

[5] Borczuk P. An evidence-based approach to the evaluation and treatment of low back pain in the 
Comparison of Routine Care Versus Transcutaneous Electrical Nerve Stimulation (TENS) for Treatment of Back Pain in the ED

emergency department. Emerg Med Pract. 2013 July;15(7):1-23.

[6] Moore RA, McQuay HJ. Prevalence of opioid adverse events in chronic non-malignant pain: systematic review of randomised trials of oral opioids. Arthritis Res Ther. 2005;7(5):R1046-51. doi: 10.1186/ar1782. Epub 2005 Jun 28. PMID: 16207320; PMCID: PMC1257433.

[7] DeSantana JM, Walsh DM, Vance C, Rakel BA, Sluka KA. Effectiveness of transcutaneous electrical nerve stimulation for treatment of hyperalgesia and pain. Curr Rheumatol Rep. 2008 Dec;10(6):492-9. doi: 10.1007/s11926-0080080-z. PMID: 19007541; PMCID: PMC2746624.

[8] Pivec R, Minshall ME, Mistry JB, Chughtai M, Elmallah RK, Mont MA. Decreased Opioid Utilization and Cost at One Year in Chronic Low Back Pain Patients Treated with Transcutaneous Electric Nerve Stimulation (TENS). Surg Technol Int. 2015 Nov; 27:268-74.

[9] Shen FH, Samartzis D, Andersson GB. Nonsurgical management of acute and chronic low back pain. J Am Acad Orthop Surg. 2006 Aug;14(8):477-87.

[10] Resende L, Merriwether E, Rampazo ÉP, et al. Meta-analysis of transcutaneous electrical nerve stimulation for relief of spinal pain. Eur J Pain. 2017 Apr; 22(4).

[11] Tang ZY, Wang HQ, Xia XL, Tang Y, Peng WW, Hu L. Mechanisms and applications of transcutaneous electrical nerve stimulation in analgesia. Sheng Li Xue Bao. 2017 Jun; 69(3):325-334.

[12] National Hospital Ambulatory Medical Care Survey: 2017 Emergency Department Summary Tables https://www.cdc.gov/nchs/data/ nhamcs/web_tables/2017_ed_web_tables-508. pdf (Accessed March 3, 2020)

[13] Friedman, BW, Chilstrom, M, Bijur, PE, Gallagher, JE. Diagnostic testing and treatment of low back pain in US emergency departments. A national perspective. Spine. 2010 Nov 15; 35(24): E1406E1411.

[14] National Institute on Drug Abuse Report. Revised January 2019. https://www.drugabuse. gov/related-topics/trends-statistics/overdosedeath-rates (accessed March 3, 2020)
[15] Scholl L, Seth P, Kariisa M, Wilson N, Baldwin G. Drug and Opioid-Involved Overdose Deaths United States, 2013-2017. MMWR Morb Mortal Wkly Rep 2019;67:1419-1427.

[16] Overdose Death Maps. Centers for Disease Control and Prevention. https://www.cdc.gov/ drugoverdose/data/prescribing/overdosedeath-maps.html. Published August 13, 2019. Accessed March 13, 2020.

[17] Hedegaard H, Chen LH, Warner M. Drugpoisoning deaths involving heroin: United States, 2000-2013. NCHS Data Brief. 2015;190:1-8.

[18] American College of Emergency Physicians: 2018 Letter to Congress https://www.acep. org/globalassets/uploads/uploaded-files/acep/ advocacy/federal-issues/letters-to-congress/ acep-response----ways-and-means-opioids-rfi---03152018.pdf (Accessed March 8, 2020)

[19] Innovative Program Targets Five Common Pain Syndromes with Non-opioid Alternatives. ED Manag. 2016 Jun;28(6):61-6.

[20] Cisewski, D, Motov, S, Essential pharmacologic options for acute pain management in the emergency setting, Turk J Emerg Med. 2019 Jan; 19(1): 1-11.

[21] Jorgensen JE, Afzali T, Riis A. Effect of differentiating exercise guidance based on a patient's level of low back pain in primary care: a mixed-methods systematic review protocol. $B M J$ Open. 2018 Jan 23; 8(1):e019742.

[22] Stochkendahl MJ, Kjaer P, Hartvigsen J, et al. National clinical guidelines for non-surgical treatment of patients with recent onset low back pain or lumbar radiculopathy. Eur Spine J. 2018;27:60-75.

[23] UK National Institute for Health and Care Excellence. Low back pain and sciatica in over $16 \mathrm{~s}$ : assessment and management. November 2016. https://www.nice.org.uk/guidance/ng59. (Accessed March 4, 2020).

[24] Qaseem A,WiltTJ,McLean RM, Forciea MA, Clinical Guidelines Committee of the American College of Physicians Noninvasive treatments for acute, subacute, and chronic low back pain: a clinical practice guideline from the American College of 
Comparison of Routine Care Versus Transcutaneous Electrical Nerve Stimulation (TENS) for Treatment of Back Pain in the ED

Physicians. Ann Intern Med. 2017;166:514-530.

[25] Vance CG, Dailey DL, Rakel BA, Sluka KA. Using TENS for pain control: the state of the evidence. Pain Manag. 2014;4(3):197-209.

[26] Grover CA, McKernan MP, Close RJH. Transcutaneous Electrical Nerve Stimulation (TENS) in the Emergency Department for Pain
Relief: A Preliminary Study of Feasibility and Efficacy. West J Emerg Med. 2018;19(5):872876.

[27] Wongrakpanich S, Wongrakpanich A, Melhado $\mathrm{K}$, Rangaswami J. A comprehensive Review of Non-Steroidal Anti-Inflammtory Drug Use in The Elderly. Aging Dis. 2018; 9(1):143-150.

\section{ARTicle Summary}

\section{Why is this topic important?}

One of the most frequent complaints of patients presenting to the ED is pain. Giving providers modalities to use, besides medications to treat pain, especially avoiding utilization of opioids is a very valuable modality.

\section{What does this study attempt to show?}

The study shows that transcutaneous electrical nerve stimulation (TENS) is a promising therapy in the ED.

\section{What are the key findings?}

TENS therapy is not inferior as a modality for back pain control in the ED, as compared to conventional therapy. TENS therapy for back pain does not have negative effect on patient throughput in the ED. 4. How is patient care impacted?

TENS units could become and additional modality for patient pain management in the ED and could be added to ALTO treatment protocols.

Citation: Yanina Purim-Shem-Tov, Jean-Philippe Daniel, Kevin Dyer, Michael Lavin, Nicholas Chien, Shital Shah. Comparison of Routine Care Versus Transcutaneous Electrical Nerve Stimulation (TENS) for Treatment of Back Pain in the ED. Archives of Emergency Medicine and Intensive Care. 2021; 4(1): 01-08. DOI: https://doi.org/10.22259/2638-5007.0401001

Copyright: (c) 2021 Yanina Purim-Shem-Tov, Jean-Philippe Daniel, Kevin Dyer, Michael Lavin, Nicholas Chien, Shital Shah. This is an open access article distributed under the Creative Commons Attribution License, which permits unrestricted use, distribution, and reproduction in any medium, provided the original work is properly cited. 